\title{
Baseline Vitamin D as a Predictor of Mortality among Hospitalized Patients with Acute Exacerbations of Chronic Obstructive Pulmonary Disease in an Endemically Vitamin D-Deficient Area in North India
}

\author{
Mohammad Ramzan Bhat, Shabir Ahmad Dar', Mir Waseem¹, Mir Nadeem² \\ SDH Sopore, ${ }^{1} \mathrm{GMC}$, Baramulla, ${ }^{2} \mathrm{GMC}$, Srinagar, Jammu and Kashmir, India
}

\section{Abstract}

Background: Conflicting data suggest that Vitamin D reduces the risk of developing chronic obstructive pulmonary disease (COPD) and COPD exacerbations. Scant data are available regarding the Vitamin D status in patients with COPD in India where Vitamin D deficiency is rampant. Objectives: We set out to study the role of baseline Vitamin D on the outcome of acute exacerbation of COPD (AECOPD) in terms of exacerbations, hospitalizations, and mortality. Materials and Methods: In a prospective observational design, 147 consenting participants with AECOPD were recruited and serum Vitamin D assessed at admission. The patients were followed for 3 months for treatment outcomes in terms of mortality, readmissions and exacerbations. Standard statistical methods were used to compare the results among those with sufficient and insufficient Vitamin D status at baseline. Results: Out of 147 completely followed participants, 113 (76.9\%) were deficient in Vitamin $\mathrm{D}$ (mean \pm standard deviation $=11.79 \pm 8.5$ [normal 30-100 ng/ml]). Patients with low Vitamin $\mathrm{D}$ were found to have a significantly higher 90-day mortality than those with normal Vitamin D status $(P=0.42$; hazard ratio $4.8, P=0.032)$. No statistically significant differences were observed in rates of exacerbations or hospital admission. Conclusion: Patients of AECOPD having baseline Vitamin D deficiency at admission have a higher mortality than those with normal Vitamin D level. Thus, Vitamin D level can serve as a prognostic indicator of the outcome of AECOPD. Larger studies are suggested to further study the association and also determine any role of Vitamin D in the treatment of such cases.

Keywords: Chronic obstructive pulmonary disease, hazard ratio, mortality, Vitamin D

\section{INTRODUCTION}

Chronic obstructive pulmonary disease (COPD) is featured by persistent airflow obstruction that is progressive and associated with an enhanced chronic inflammatory response in the airways and the lung to noxious particles or gases and is amenable to prevention and treatment. COPD, a disease of mounting public health importance around the globe, is currently the fourth leading cause of death and estimates suggest that COPD will figure at the third most common cause of death worldwide by $2020 .{ }^{[1]}$ The prevalence of chronic airflow limitation in Kashmir, North India, that has temperate geography with severe winters is high with $17.3 \%$ in males and $14.8 \%$ in females. ${ }^{[2]}$

The natural history of COPD apart from an inexorable decline in lung function, is punctuated by exacerbations which are

\begin{tabular}{|l|l|}
\hline \multicolumn{2}{|c|}{ Access this article online } \\
\hline Quick Response Code: & Website: \\
\hline & www.ijrconline.org \\
\hline & \\
\hline
\end{tabular}

catastrophic events leading to excessive morbidity, high mortality, accelerated decline of lung function and a high economic cost. We have demonstrated recently that acute exacerbation in COPD (AECOPD) has a high 2-year mortality such that appropriate strategies aimed at reducing the frequency and severity of exacerbations are warranted ${ }^{[3]}$ During exacerbations of COPD, mortality is high $(39 \%)^{[3]}$ with a high correlation to per Capita Gross National Income with adjusted potential cofounders. ${ }^{[2]}$

Address for correspondence: Dr. Mir Nadeem C/O Dr Mir Waseem, Doctors' Quarters, GMC, Baramulla, Kantbagh, Baramulla - 193 101, Jammu and Kashmir, India. E-mail: mirnadeem44@gmail.com

This is an open access journal, and articles are distributed under the terms of the Creative Commons Attribution-NonCommercial-ShareAlike 4.0 License, which allows others to remix, tweak, and build upon the work non-commercially, as long as appropriate credit is given and the new creations are licensed under the identical terms.

For reprints contact: reprints@medknow.com

How to cite this article: Bhat MR, Dar SA, Waseem M, Nadeem M. Baseline Vitamin D as a predictor of mortality among hospitalized patients with acute exacerbations of chronic obstructive pulmonary disease in an endemically Vitamin D-deficient area in North India. Indian J Respir Care 2020;9:88-93.

Received: 19-09-2019 Revised: 27-11-2019

Accepted: 02-12-2019 Published: 08-01-2020 
While the importance of Vitamin D in bone health has been recognized for centuries, there has been recent interest in the extra-skeletal roles for Vitamin D including lung disorders. ${ }^{[4]}$ There is association of Vitamin D insufficiency with increased risk and severity of respiratory infections. ${ }^{[5]}$ The high prevalence of osteoporosis and osteopenia in COPD patients may be an indication of a link between Vitamin $\mathrm{D}$ deficiency and COPD. ${ }^{[4]}$ Vitamin D can be beneficial in reducing exacerbations when taken in adequate amount and for a prolonged period of time. ${ }^{[6]}$

Previous studies on Vitamin D and COPD have recorded conflicting results and no data are available from the Indian subcontinent. Vitamin D deficiency is common in Kashmir (Indian administered); ${ }^{[7]}$ however, scant data are available on the prevalence of Vitamin D deficiency in COPD patients especially those with exacerbations and the influence, if any, on the outcomes of the exacerbations. The current study was designed against this backdrop to determine the baseline Vitamin D status of patients hospitalized with AECOPD in a high prevalence area for COPD with endemic Vitamin D deficiency and the effect of baseline Vitamin D status on 90-day mortality is such cases.

\section{Materials and Methods}

Hundred and forty-seven consecutive consenting patients with AECOPD who presented to the emergency room (ER) of a 850-bedded tertiary care cum referral center of North India from June 2015 to May 2016, and required hospitalization were recruited for the study.

After recruitment, clinical history and examination were undertaken in all patients and recorded on a predefined case record form. History and patterns of smoking, use of any medications, history of exacerbations, comorbidities, and history of previous Vitamin D administration were specifically sought.

All patients underwent routine hematological and biochemical investigations. Radiography of the chest, electrocardiogram, sputum examination, and ultrasound of the chest/abdomen were obtained in all. Computed tomography of the chest and other investigations were performed as appropriate. Serial arterial blood gases were obtained in all patients for monitoring progress on treatment.

3-5 ml blood was collected in serum vial and kept at room temperature for half an hour. After the incubation, serum was separated by centrifuging the tubes at $2000 \mathrm{rpm}$ for $5 \mathrm{~min}$. Serum was transferred into $1.5 \mathrm{ml}$ Eppendorf tubes and stored at $-80^{\circ} \mathrm{C}$. Vitamin $\mathrm{D}$ estimation was done by chemiluminescence method.

The patients were treated using standard protocols including bronchodilators, oxygen, noninvasive/invasive ventilation, antibiotics, steroids, antivirals (if appropriate), and other supportive measures. Daily monitoring was performed and patients discharged once stabilized. The patients were advised to follow in the outpatient department on a weekly basis and post discharge follow-up was completed by telephonic surveys. Requirement for readmission or mortality was recorded. Followups were completed telephonically or by a personal home visit.

Written informed consent was taken from all study participants. The study was approved by PG Institutional Ethical Committee, Sher-i-Kashmir Institute of Medical Sciences, Srinagar.

\section{Definitions and exclusions}

COPD was defined as a postbronchodilator spirometry with an forced expiratory volume in $1 \mathrm{~s}\left(\mathrm{FEV}_{1}\right)$ /forced vital capacity (FVC) ratio of $<0.7$ and staging was based on $\mathrm{FEV}_{1}$ ratio into Stages I, II, III, and IV as per GOLD criteria. Frequent exacerbations were defined as having $\geq 2$ exacerbations treated with antibiotics and/or oral steroids and/or hospitalization in the last 12 months. ${ }^{[1]}$

Patients having tuberculosis, primary pulmonary hypertension, pulmonary embolism, restrictive airway disease, conditions associated with Vitamin D metabolism, absorption or taking Vitamin D containing medications, and known autoimmune diseases or any active cancer in the last 5 years were excluded from the study.

\section{Statistical methods}

All analyses were performed on SPSS Version 20.0 (IBM Corp., IBM SPSS Statistics for Windows, Armonk, NY, USA). Student's independent $t$-test or Mann-Whitney U-test, whichever appropriate, was employed for comparing continuous variables. Chi-square test or Fisher's exact test, whichever appropriate, was applied for comparing categorical variables. Kaplan-Meier survival analysis and Cox regression analysis were performed to compare the survival pattern of patients with low and normal Vitamin D levels. $P<0.05$ was considered as statistically significant.

\section{RESULTS}

The 147 recruited patients included 78 males and 69 females with ages ranging from 38 to 91 years (median 71 years) with the majority being aged $>50$ years [Table 1]. A history of ever smoking was elicitable in $68 \%$ of the participants, hookah smoking being the most common form of smoking. Other demographic features are summarized in Table 1.

Table 2 shows the baseline Vitamin D levels of the study participants. The mean value of Vitamin D was $23.61 \pm 28.773 \mathrm{ng} /$ $\mathrm{ml}$ (range $=1.11-155.87)$. Majority, i.e. $113(76.9 \%)$ of the individuals had low levels of Vitamin D, 29 (19.7\%) had normal levels of Vitamin D, and a small number, i.e. 5 (3.4\%) had high levels of Vitamin D with mean \pm standard deviation of $11.79 \pm 8.565$ (range $=1.11-29.53) \mathrm{ng} / \mathrm{ml}$, $50.07 \pm 19.328($ range $=31.12-99.59) \mathrm{ng} / \mathrm{ml}$, and $137.19 \pm 19.994$ $($ range $=115.37-155.87) \mathrm{ng} / \mathrm{ml}$, respectively, as shown in Table 2 .

At the end of 3 months, 108 (73.47\%) patients were alive and $39(26.53 \%)$ had expired as shown in Figure 1. Those 
Bhat, et al.: Vitamin D levels and mortality prediction in COPD

\begin{tabular}{|c|c|c|c|}
\hline & \multicolumn{2}{|c|}{ Vitamin D } & \multirow[t]{2}{*}{$P$} \\
\hline & Low (\%) & Normal (\%) & \\
\hline \multicolumn{4}{|l|}{ Age (years) } \\
\hline $36-50$ & $6(100.0)$ & $0(0.0)$ & 0.800 \\
\hline $51-65$ & $41(78.8)$ & $11(21.2)$ & \\
\hline $66-80$ & $49(77.8)$ & $14(22.2)$ & \\
\hline$>80$ & $17(81.0)$ & $4(19.0)$ & \\
\hline \multicolumn{4}{|l|}{ Gender } \\
\hline Male & $62(83.8)$ & $12(16.2)$ & 0.195 \\
\hline Female & $51(75.0)$ & $17(25.0)$ & \\
\hline \multicolumn{4}{|l|}{ Smoking status } \\
\hline Current smoker & $14(82.4)$ & $3(17.6)$ & 0.956 \\
\hline Ex-smoker & $61(78.2)$ & $17(21.8)$ & \\
\hline Nonsmoker & $38(80.9)$ & $9(19.1)$ & \\
\hline \multicolumn{4}{|l|}{ Smoking type } \\
\hline Cigarette & $13(76.5)$ & $4(23.5)$ & 0.902 \\
\hline Huka & $57(79.2)$ & $15(20.8)$ & \\
\hline Both & $5(83.3)$ & $1(16.7)$ & \\
\hline \multicolumn{4}{|l|}{ BMI $\left(\mathrm{kg} / \mathrm{m}^{2}\right)$} \\
\hline$<18.5$ & $2(100.0)$ & $0(0.0)$ & 0.762 \\
\hline $18.50-24.99$ & $65(77.4)$ & $19(22.6)$ & \\
\hline $25.00-29.99$ & $37(84.1)$ & $7(15.9)$ & \\
\hline$\geq 30$ & $9(75.0)$ & $3(25.0)$ & \\
\hline \multicolumn{4}{|l|}{ GOLD staging } \\
\hline 2 & $37(77.1)$ & $11(22.9)$ & 0.868 \\
\hline 3 & $50(80.6)$ & $12(19.4)$ & \\
\hline 4 & $26(81.2)$ & $6(18.8)$ & \\
\hline
\end{tabular}

\begin{tabular}{|c|c|c|c|}
\hline Vitamin D & $n(\%)$ & Mean \pm SD & Range \\
\hline Low $(<30 \mathrm{ng} / \mathrm{ml})$ & $113(76.9)$ & $11.79 \pm 8.565$ & $1.11-29.53$ \\
\hline Normal (30-100 ng/ml) & $29(19.7)$ & $50.07 \pm 19.328$ & $31.12-99.59$ \\
\hline $\operatorname{High}(>100 \mathrm{ng} / \mathrm{ml})$ & $5(3.4)$ & $137.19 \pm 19.994$ & $115.37-155.87$ \\
\hline Total & $147(100)$ & $23.61 \pm 28.773$ & $1.11-155.87$ \\
\hline
\end{tabular}

SD: Standard deviation

who expired, i.e., $15(10.2 \%)$ died during hospital stay and $24(16.3 \%)$ died at home after discharge from the hospital. Those who expired during hospital stay were admitted with frequent exacerbations of COPD and type 2 respiratory failure. We were not able to ascertain the actual cause of death in $16.3 \%$ of patients who died at home but on telephonic communication, it was established that most of patients died of respiratory symptoms or had a sudden death.

On univariate analysis [Tables 3 and 4], a trend was observed toward a higher mortality in those aged $<80$ years compared to those aged $>80$ years; however, the difference was not statistically significant. There was gender-based difference in survival, but a significant difference of survival was observed among ever smokers versus nonsmokers. No differences in survival were observed based on the GOLD stage of the patients. Differences in survival were observed between those

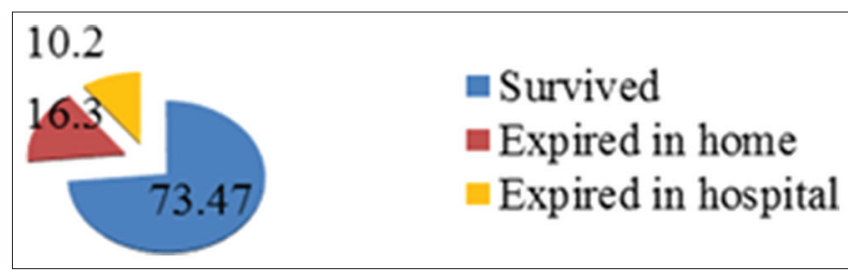

Figure 1: Outcome at 3 months

with a normal serum Vitamin D level compared to those with a low Vitamin D levels $(93.1 \%$ vs. $70.8 \%$, respectively, odds ratio $[\mathrm{OR}] 0.18, P=0.014)$. After exclusion of the patients with high Vitamin D from analysis, mean Vitamin D levels were higher among those who survived compared to those who did not [Table 4].

Hospital readmissions ( $>2)$ were observed in $35(23.8 \%)$ cases. There were no statistical differences between Vitamin D levels among those with hospital readmissions ( $>2)$, and the frequency of exacerbations was similar among those with lower and normal Vitamin D levels. Hospital readmissions $(\geq 2)$ were found in $23.9 \%, 20.7 \%$, and $40.0 \%$ of the participants with low, normal, and high levels of Vitamin D levels, respectively. Mean levels of Vitamin D were not statistically different among those with exacerbations $(\geq 2)$ compared to those with $(\leq 2)$ exacerbations $(27.20+2.6$ versus $33.3+5.6, P=0.28)$.

Figure 2 depicts Kaplan-Meier estimator showing two curves for normal and low levels of Vitamin D. Participants with low levels of Vitamin D had a survival of $70.8 \%$ against $93.1 \%$ survival of the participants with normal levels of Vitamin D. Overall survival of $75.9 \%$ was seen at 3 -month follow-up. The mean survival time in weeks was 9.6 (confidence interval [CI] $=8.879-10.325)$ in those who died in low Vitamin D group against $11.5(\mathrm{CI}=10.789-12.176)$ in those who died in normal Vitamin D group.

Multivariate proportional hazards analysis for Vitamin D is shown in Figure 2. Those participants who had low Vitamin D had mortality hazard ratio of 4.789 (1.149-19.961) as compared to the study participants having normal Vitamin D.

\section{Discussion}

Our data show that patients with Vitamin D level of $<30$ have a higher 90 -day mortality $(29.2 \%)$ compared to those with normal Vitamin D levels (6.9\%) with a hazard ratio for mortality as $4.789(95 \% \mathrm{CI}=1.149-19.961)$. This study opens up newer vistas for possible intervention in AECOPD in our endemically Vitamin D-deficient population.

In this study, we have focused on the outcome of A/E of COPD viz a viz baseline Vitamin D levels with the assumption that decreased Vitamin D levels adversely affect the outcome of $\mathrm{A} / \mathrm{E}$ of COPD. However, we could not find any statistical significance between Vitamin D levels and number of exacerbations and hospitalizations with $P(0.633$ and 1.00$)$, respectively; same observations were made by study done by Lehouck et al. 2012. ${ }^{[8]}$ They also tried to explore whether 
Bhat, et al.: Vitamin D levels and mortality prediction in COPD

\begin{tabular}{|c|c|c|c|c|}
\hline & \multicolumn{2}{|c|}{ Outcome } & \multirow[t]{2}{*}{ OR (95\% Cl) } & \multirow[t]{2}{*}{$P$} \\
\hline & Survived, $n$ (\%), 108 (73.5\%) & Expired, $n(\%), 39$ (26.5\%) & & \\
\hline \multicolumn{5}{|l|}{ Age (years) } \\
\hline $36-50$ & $5(83.3)$ & $1(16.7)$ & $0.833(0.070-9.858)$ & 1.000 \\
\hline $51-65$ & $39(72.2)$ & $15(27.8)$ & $0.433(0.111-1.687)$ & 0.366 \\
\hline $66-80$ & $46(69.7)$ & $20(30.3)$ & $0.383(0.101-1.449)$ & 0.169 \\
\hline$>80$ & $18(85.7)$ & $3(14.3)$ & Constant & \\
\hline \multicolumn{5}{|l|}{ Gender } \\
\hline Male & $58(74.4)$ & $20(25.6)$ & $1.102(0.529-2.294)$ & 0.853 \\
\hline Female & $50(72.5)$ & $19(27.5)$ & & \\
\hline \multicolumn{5}{|l|}{ Smoking status } \\
\hline Current smoker & $16(84.2)$ & $3(15.8)$ & $1.094(0.256-4.658)$ & 1.000 \\
\hline Ex-smoker & $53(65.4)$ & $28(34.6)$ & $0.388(0.159-0.943)$ & 0.033 \\
\hline Nonsmoker & $39(83.0)$ & $8(17.0)$ & Constant & \\
\hline \multicolumn{5}{|l|}{ GOLD staging } \\
\hline 2 & $36(72.0)$ & $14(28.0)$ & $0.857(0.312-2.354)$ & 0.764 \\
\hline 3 & $48(73.8)$ & $17(26.2)$ & $0.941(0.355-2.489)$ & 0.902 \\
\hline 4 & $24(75.0)$ & $8(25.0)$ & Constant & \\
\hline \multicolumn{5}{|l|}{ Vitamin D* $\mathrm{ng} / \mathrm{ml}$} \\
\hline Low $(<30)$ & $80(70.8)$ & $33(84.6 \%)$ & $0.179(0.040-0.798)$ & 0.014 \\
\hline Normal (30-100) & $27(93.1)$ & $2(6.9)$ & & \\
\hline
\end{tabular}

\section{Table 4: Unpaired $t$-test for different variables on admission versus outcome}

\begin{tabular}{lccc}
\hline & \multicolumn{2}{c}{ Mean \pm SD (range) } & $P$ \\
\cline { 2 - 3 } & Survived $(\boldsymbol{n = 1 0 8 )}$ & Expired $(\boldsymbol{n}=\mathbf{3 9})$ & \\
\hline Age & $68.12 \pm 10.26(41-85)$ & $67.20 \pm 10.06(38-91)$ & 0.632 \\
$\mathrm{FEV}_{1}$ & $45.53 \pm 14.70(21-115)$ & $47.59 \pm 18.04(18-83)$ & 0.482 \\
$\mathrm{FVC}$ & $70.96 \pm 53.62(38-113)$ & $70.40 \pm 20.97(42-121)$ & 0.950 \\
$\mathrm{FEV}_{1} / \mathrm{FVC}$ & $1.51 \pm 0.26(0.69-1.70)$ & $1.52 \pm 0.32(0.63-1.70)$ & 0.804 \\
$\mathrm{BMI}_{\text {Vitamin }}$ D $^{*}$ & $24.99 \pm 3.42(17.7-36.7)$ & $23.93 \pm 3.27(18.3-35.1)$ & 0.095 \\
& $21.82 \pm 21.02$ & $12.83 \pm 10.20$ & 0.016 \\
& $(1.11-99.59)$ & $(1.91-45.17)$ & $0.042^{* *}$ \\
\hline
\end{tabular}

*5 hypervitaminosis cases excluded from analysis, **Mann-Whitney U-test used for $P$ value calculation. $\mathrm{FEV}_{1}$ : Forced expiratory volume in 1s, FVC: Forced vital capacity, BMI: Body mass index, SD: Standard deviation

supplementation with high doses of Vitamin D could reduce the incidence of COPD exacerbations. They designed a randomized, single-center, double-blind, placebo-controlled trial. 182 patients with moderate to very severe COPD and a history of recent exacerbations were studied. The median time to first exacerbation did not significantly differ between the groups (hazard ratio, 1.1 [CI, 0.82-1.56]; $P=0.41$ ), nor did exacerbation rates, $\mathrm{FEV}_{1}$, hospitalization, quality of life, and death. ${ }^{[8]}$

Mekov et al. in their study found relatively low 1-year mortality in COPD patients after surviving severe exacerbation. They also noticed that Grade $\mathrm{C}$ and $\mathrm{FEV}_{1}>80 \%$ may be factors for good prognosis, while age, $\mathrm{FEV}_{1}$ value, severe exacerbation in previous year, and reduced quality of life were various risk factors for increased mortality. Cox regression showed

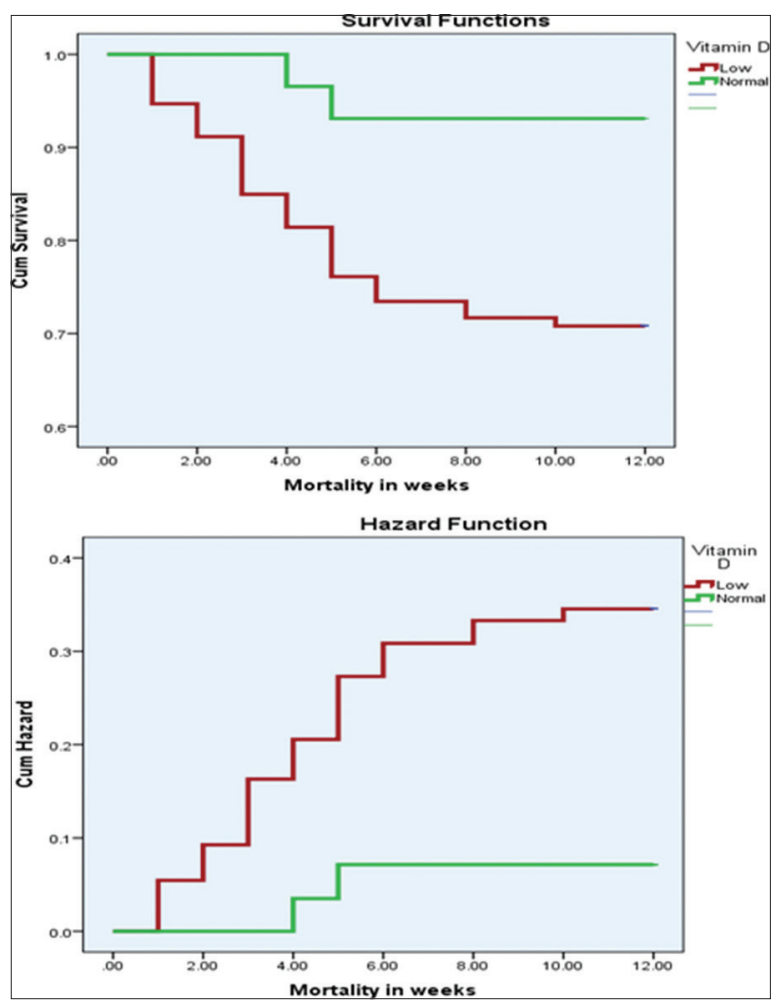

Figure 2: Kaplan-Meier survival analysis

that Vitamin D levels are not predictors for mortality. ${ }^{[9]} \mathrm{Zhu}$ et al. in a systemic review and meta-analysis concluded that severe Vitamin D deficiency is associated with increased risk of COPD and severe COPD but not with COPD exacerbation. This meta-analysis showed that lower serum Vitamin D levels were found in COPD patients than in controls (standardized 
mean difference (SMD) $-0.69,95 \% \mathrm{CI}, P<0.001)$ especially in severe COPD (SMD: $-0.87,95 \% \mathrm{CI} ; P=0.001)$ and with COPD severity (OR: 2.83, 95\% CI $P<0.001$ ) but not with COPD exacerbation (OR; $1.17,95 \%$ CI $P=0.326) .{ }^{[10]}$ Our study also did not demonstrate a higher risk of exacerbations or hospitalization in patients who were Vitamin D deficient compared to those who were having normal Vitamin D levels. However, Khan et al. concluded that when given for prolonged period, Vitamin D supplementation has significant effect in reducing number of AECOPD patients. ${ }^{[11]}$

A recent study among Indian patients with COPD concluded that COPD is associated with a significantly low level of Vitamin D that was independent of patients' age, gender, BMI, and residence. The lower level of Vitamin $\mathrm{D}$ was associated with severity of COPD, increased frequency of A/E COPD and increased frequency of hospitalization. The relationship between lung functions and levels of vit D was almost linear. ${ }^{[12]}$ Our study was, however, not designed to address this outcome in Vitamin D-deficient participants.

Patients with COPD are at high risk for Vitamin D deficiency because they are more prone to skin aging (due to smoking), have reduced outdoor activity, exhibit reduced food intake, and are often treated with corticosteroids which increases Vitamin D catabolism. Epidemiological studies in healthy subjects have reported a strong relationship between 25 -hydroxyvitamin D serum levels and pulmonary function, as assessed by $\mathrm{FEV}_{1}$ and FVC. ${ }^{[13]}$

Among many environmental and genetic factors, the role of various nutrients, especially Vitamin D in COPD has been studied by various researchers. Studies conducted so far showing the impact of Vitamin D deficiency on the outcome of COPD have shown mixed results in terms of mortality and morbidity. ${ }^{[8,14-20]}$ While findings from Monadi et al ${ }^{[15]}$ indicated a relationship between serum Vitamin $\mathrm{D}$ levels and $\mathrm{FEV}_{1}$ volume in COPD patients, Malinovschi et al. ${ }^{[17]}$ concluded that severe Vitamin D deficiency was associated with more frequent COPD exacerbations and hospitalization. Further Lehouck et al ${ }^{[8]}$ and Martineau et al.$^{[19]}$ findings suggested that correction of Vitamin D deficiency in COPD patients reduces the risk of exacerbations.

However, there is definite mortality benefit in patients with normal levels of Vitamin D. We had 147 individuals for analysis; 5 cases were excluded in view of hypervitaminosis. The individuals who survived had higher mean Vitamin D levels compared to those expired with the difference statistically significant. Similar observation was made by Lee et al. 2014. ${ }^{[16]}$

Controlled trials examining the supplementation of Vitamin D on reducing the respiratory tract infections (RTI's) have shown mixed results with some decreasing frequency of RTI and favoring supplementation while others have not demonstrated the same; ${ }^{[21-31]}$ however, cumulative benefits of Vitamin D being anti-inflammatory, antiautoimmune, antiangiogenic and anticancer, antidepressant ${ }^{[32]}$ makes its deficiency compelling for the treating physician to get corrected. Advanced age and limited mobility limit the sun exposure of COPD patients, especially in cold areas with poor winter sun is likely to have an exacerbating effect on the Vitamin D status of this endemically Vitamin D-deficient area. Although there are other compelling reasons for treating Vitamin D deficiency in such cases, our data argue for another possible reason for addressing the Vitamin D-deficient status of such cases. However, the influence on the outcome of exacerbations needs be studied through carefully designed placebo-controlled studies.

\section{Conclusion}

Patients of AECOPD having baseline Vitamin D deficiency at admission have a higher mortality than those with normal Vitamin D level. Thus, Vitamin D level can serve as a prognostic indicator of the outcome of AECOPD. Larger studies are suggested to further study the association and also determine any role of Vitamin D in the treatment of such cases. Nonetheless Vitamin D supplementation in patients with demonstrable deficiency would certainly be of value regardless of its effect on the outcome of COPD whether it helps a favorable outcome needs further study.

\section{Financial support and sponsorship}

Nil.

\section{Conflicts of interest}

There are no conflicts of interest.

\section{RefERENCES}

1. Fabbri LM, Hurd SS; GOLD Scientific Committee. Global strategy for the diagnosis, management and prevention of COPD: 2003 update. Eur Respir J 2003;22:1-2.

2. Burney $\mathrm{P}$, Jithoo A, Kato B, Janson C, Mannino D, Niankowska-Mogilnicka E, et al. Chronic obstructive pulmonary disease mortality and prevalence: The associations with smoking and poverty-a BOLD analysis. Thorax 2014;69:465-73.

3. Koul PA, Dar HA, Jan RA, Shah S, Khan UH. Two-year mortality in survivors of acute exacerbations of chronic obstructive pulmonary disease: A North Indian study. Lung India 2017;34:511-6.

4. Foong RE, Zosky GR. Vitamin D deficiency and the lung: Disease initiator or disease modifier? Nutrients 2013;5:2880-900.

5. Koul PA. Vitamin D and respiratory infections J Med Med Sci 2012;3:83-6.

6. Khan DM. Role of Vitamin D in reducing number of acute exacerbations in COPD patients. J Med Sci 2017;33:610-4.

7. Zargar AH, Ahmad S, Masoodi SR, Wani AI, Bashir MI, Laway BA, et al. Vitamin D status in apparently healthy adults in Kashmir Valley of Indian subcontinent. Postgrad Med J 2007;83:713-6.

8. Lehouck A, Mathieu C, Carremans C, Baeke F, Verhaegen J, Van Eldere J, et al. High doses of Vitamin D to reduce exacerbations in chronic obstructive pulmonary disease: A randomized trial. Ann Intern Med 2012;156:105-14.

9. Mekov E, Slavova Y, Tsakova A, Genova MP, Kostadinov DT, Minchev D, et al. One-year mortality after severe COPD exacerbation in Bulgaria. PeerJ 2016;4:e2788.

10. Zhu M, Wang T, Wang C, Ji Y. The association between vitamin D and COPD risk, severity, and exacerbation: An updated systematic review and meta-analysis. Int J Chron Obstruct Pulmon Dis 2016;11:2597-607.

11. Khan DM, Ullah A, Randhawa FA, Iqtadar S, Butt NF, Waheed K. Role of Vitamin D in reducing number of acute exacerbations in chronic 
Bhat, et al.: Vitamin D levels and mortality prediction in COPD

obstructive pulmonary disease (COPD) patients. 2017;33:610-4.

12. Gupta BK, Meena SL, Saini M, Nehara HR, Saini ML, Gupta J. Evaluation of Vitamin-D level in patients of chronic obstructive pulmonary disease (COPD) and its clinical correlation. Innov J Med Health Sci 2016;3:107-12.

13. Janssens W, Bouillon R, Claes B, Carremans C, Lehouck A, Buysschaert I, et al. Vitamin D deficiency is highly prevalent in COPD and correlates with variants in the Vitamin D-binding gene. Thorax 2010;65:215-20.

14. Hornikx M, Van Remoortel H, Lehouck A, Mathieu C, Maes K, Gayan-Ramirez G, et al. Vitamin D supplementation during rehabilitation in COPD: A secondary analysis of a randomized trial. Respir Res 2012;13:84.

15. Monadi M, Heidari B, Asgharpour M, Firouzjahi A, Monadi M, Ghazi Mirsaied MA. Relationship between serum Vitamin D and forced expiratory volume in patients with chronic obstructive pulmonary disease (COPD). Caspian J Intern Med 2012;3:451-5.

16. Lee HM, Liu M, Lee K, Luo Y, Wong ND. Does low Vitamin D amplify the association of COPD with total and cardiovascular disease mortality? Clin Cardiol 2014;37:473-8.

17. Malinovschi A, Masoero M, Bellocchia M, Ciuffreda A, Solidoro P, Mattei A, et al. Severe Vitamin D deficiency is associated with frequent exacerbations and hospitalization in COPD patients. Respir Res 2014; $15: 131$.

18. Puhan MA, Siebeling L, Frei A, Zoller M, Bischoff-Ferrari H, Ter Riet G. No association of 25-hydroxyvitamin D with exacerbations in primary care patients with COPD. Chest 2014;145:37-43.

19. Martineau AR, James WY, Hooper RL, Barnes NC, Jolliffe DA, Greiller CL, et al. Vitamin D3 supplementation in patients with chronic obstructive pulmonary disease (ViDiCO): A multicentre, double-blind, randomised controlled trial. Lancet Respir Med 2015;3:120-30.

20. Said AF, Abd-Elnaeem EA. Vitamin D and chronic obstructive pulmonary disease. Egypt J Chest Dis Tuberc 2015;64:67-73.

21. Avenell A, Cook JA, Maclennan GS, Macpherson GC. Vitamin D supplementation to prevent infections: A sub-study of a randomised placebo-controlled trial in older people (RECORD trial, ISRCTN
51647438). Age Ageing 2007;36:574-7.

22. Li-Ng M, Aloia JF, Pollack S, Cunha BA, Mikhail M, Yeh J, et al. A randomized controlled trial of Vitamin D3 supplementation for the prevention of symptomatic upper respiratory tract infections. Epidemiol Infect 2009;137:1396-404.

23. Vieth R, Chan PC, MacFarlane GD. Efficacy and safety of Vitamin D3 intake exceeding the lowest observed adverse effect level. Am J Clin Nutr 2001;73:288-94.

24. Barger-Lux MJ, Heaney RP, Dowell S, Chen TC, Holick MF. Vitamin $\mathrm{D}$ and its major metabolites: Serum levels after graded oral dosing in healthy men. Osteoporos Int 1998;8:222-30.

25. Laaksi I, Ruohola JP, Tuohimaa P, Auvinen A, Haataja R, Pihlajamäki H, et al. An association of serum Vitamin D concentrations and $40 \mathrm{nmol} / \mathrm{L}$ with acute respiratory tract infection in young Finnish men. Am J Clin Nutr 2007;86:714-7.

26. Roth DE, Shah R, Black RE, Baqui AH. Vitamin D status and acute lower respiratory infection in early childhood in Sylhet, Bangladesh. Acta Paediatr 2010;99:389-93.

27. Wayse V, Yousafzai A, Mogale K, Filteau S. Association of subclinical Vitamin D deficiency with severe acute lower respiratory infection in Indian children under 5 y. Eur J Clin Nutr 2004;58:563-7.

28. Karatekin G, Kaya A, Salihoğlu O, Balci H, Nuhoğlu A. Association of subclinical Vitamin D deficiency in newborns with acute lower respiratory infection and their mothers. Eur J Clin Nutr 2009;63:473-7.

29. McNally JD, Leis K, Matheson LA, Karuananyake C, Sankaran K, Rosenberg AM. Vitamin D deficiency in young children with severe acute lower respiratory infection. Pediatr Pulmonol 2009;44:981-8.

30. Roth DE, Jones AB, Prosser C, Robinson JL, Vohra S. Vitamin D status is not associated with the risk of hospitalization for acute bronchiolitis in early childhood. Eur J Clin Nutr 2009;63:297-9.

31. Ginde AA, Mansbach JM, Camargo CA Jr. Association between serum 25-hydroxyvitamin D level and upper respiratory tract infection in the Third National Health and Nutrition Examination Survey. Arch Intern Med 2009;169:384-90.

32. Holick MF. Vitamin D deficiency. N Engl J Med 2007;357:266-81. 